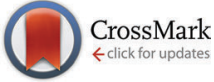

Cite this: Phys. Chem. Chem. Phys., 2016, 18, 13993

Received 26th February 2016, Accepted 25th April 2016

DOI: $10.1039 / c 6 c p 01353 h$

www.rsc.org/pccp

\title{
Structural, thermal, dielectric and phonon properties of perovskite-like imidazolium magnesium formate $\dagger$
}

\author{
Mirosław Mączka, ${ }^{\star a}$ Nathalia Leal Marinho Costa, ${ }^{\mathrm{b}}$ Anna Gągor, ${ }^{\mathrm{a}}$ \\ Waldeci Paraguassu, ${ }^{\mathrm{b}}$ Adam Sieradzki ${ }^{\mathrm{C}}$ and Jerzy Hanuza
}

\begin{abstract}
We report the synthesis and characterisation of a magnesium formate framework templated by protonated imidazole. Single-crystal $X$-ray diffraction data showed that this compound crystallizes in the monoclinic structure in the $P 2_{1} / n$ space group with lattice parameters $a=12.1246(4) \AA, b=12.2087(5) \AA, c=12.4991(4) \AA$ and $\beta=91.39(1)^{\circ}$. The antiparallel arrangement of the dipole moments associated with imidazolium cations suggests the antiferroelectric character of the room-temperature phase. The studied compound undergoes a structural phase transition at $451 \mathrm{~K}$ associated with a halving of the $c$ lattice parameter and the disappearance of the antiferroelectric order. The monoclinic symmetry is preserved and the new metrics are $a=12.261(7) \AA, b=12.290(4) \AA, c=6.280(4) \AA$, and $\beta=90.62(5)^{\circ}$. Raman and IR data are consistent with the $\mathrm{X}$-ray diffraction data. They also indicate that the disorder of imidazolium cations plays a significant role in the mechanism of the phase transition. Dielectric data show that the phase transition is associated with a relaxor nature of electric ordering. We also report high-pressure Raman scattering studies of this compound that revealed the presence of two pressure-induced phase transitions near 3 and $7 \mathrm{GPa}$. The first transition is most likely associated with a rearrangement of the imidazolium cations without any significant distortion of these cations and the magnesium formate framework, whereas the second transition leads to strong distortion of both the framework and imidazolium cations. High-pressure data also show that imidazolium magnesium formate does not show any signs of amorphization up to $11.4 \mathrm{GPa}$.
\end{abstract}

\section{Introduction}

Metal-organic framework (MOF) compounds have been widely studied in recent years due to their useful properties for wide

\footnotetext{
${ }^{a}$ Institute of Low Temperature and Structure Research, Polish Academy of Sciences, Box 1410, 50-950 Wroctaw 2, Poland. E-mail: m.maczka@int.pan.wroc.pl

${ }^{b}$ Faculdade de Física, Universidade Federal do Pará, 66075-110, Belém, PA, Brazil

${ }^{c}$ Department of Experimental Physics, Wroctaw University of Technology, Wybrzeże Wyspiańskiego 27, 50-370, Wrockaw, Poland

${ }^{d}$ Department of Bioorganic Chemistry, Faculty of Industry and Economics, Wroctaw University of Economics, 118/120 Komandorska Str., 53-345 Wroctaw, Poland $\dagger$ Electronic supplementary information (ESI) available: Tables S1-S5: crystal data for ImMg, IR and Raman wavenumber for the low- and high-temperature phases of ImMg together with the proposed assignment, and wavenumber intercepts at zero pressure and pressure coefficients for ambient and high-pressure phases of ImMg. Fig. S1-S15: DSC traces for ImMn and ImMg, heat capacity and entropy for ImMn, figure showing the presence of two symmetry-independent imidazolium cations in the perovskite-like cavities, the reciprocal lattice for ImMg, La Bail fits for low- and high-temperature phases of ImMn and ImMg, temperaturedependent IR and Raman spectra, wavenumber vs. pressure plots of the Raman modes observed in the ImMg crystal for compression experiment, high-pressure Raman spectra of ImMg recorded during the decompression experiment. CCDC 1455552. For ESI and crystallographic data in CIF or other electronic format see DOI: $10.1039 / \mathrm{c} 6 \mathrm{cp} 01353 \mathrm{~h}$
}

range of applications. ${ }^{1,2}$ Dense MOFs constitute a subclass of MOFs, in which metal nodes are connected by small organic ligands, such as $\mathrm{N}_{3}^{-}, \mathrm{CN}^{-}, \mathrm{HCOO}^{-}$or $\left[\mathrm{N}(\mathrm{CN})_{2}\right]^{-}$, forming threedimensional frameworks with cavities occupied by $\mathrm{NH}_{4}{ }^{+}$or protonated amines. ${ }^{3-5}$ Among these compounds, azides and cyanides possess interesting switchable dielectric properties, ${ }^{4,5}$ whereas tetrapropylammonium manganese dicyanamide exhibits both antiferroelectric and magnetic order in one phase. ${ }^{6}$ Most widely studied were, however, $\mathrm{AB}(\mathrm{HCOO})_{3}$ formates with $\mathrm{A}=\mathrm{NH}_{4}{ }^{+}$or protonated amine and $\mathrm{B}=\mathrm{Mg}^{2+}, \mathrm{Cd}^{2+}, \mathrm{Zn}^{2+}, \mathrm{Mn}^{2+}$, $\mathrm{Co}^{2+}, \mathrm{Fe}^{2+}, \mathrm{Ni}^{2+}$, and $\mathrm{Cu}^{2+}$, due to their rich structural diversity and the corresponding dielectric, magnetic, multiferroic, ferroelastic, mechanical, luminescent, negative thermal expansion and negative compressibility properties. ${ }^{7-23}$

In the family of metal formate frameworks, the protonated amines are located in the cavities and they form hydrogen bonds to the anionic framework. ${ }^{7,9,13,19-21}$ At room or elevated temperatures, these interactions are usually too weak to overcome the thermally activated reorientational motion of the protonated amine and, as a result, the structures are dynamically disordered. ${ }^{7,9,13,19-21}$ Strengthening of the hydrogen-bonding interactions at lower temperatures often leads to ordering of these 
cations, distortion of the framework and the appearance of ferroelectric or antiferroelectric order. ${ }^{7,9,13,19-21}$ The strength of the hydrogen bonds and thus the phase transition temperature and dielectric properties, can also be modified by changing either protonated amine or metal cation. For instance, it was reported that the replacement of $\mathrm{B}^{2+}(\mathrm{B}=\mathrm{Mn}, \mathrm{Fe}, \mathrm{Co}, \mathrm{Zn}$, and $\mathrm{Ni})$ with $\mathrm{Mg}^{2+}$ in the family of $\left[\left(\mathrm{CH}_{3}\right)_{2} \mathrm{NH}_{2}\right]\left[\mathrm{B}(\mathrm{HCOO})_{3}\right]$ formates leads to a pronounced increase in the phase transition temperature from $160-185 \mathrm{~K}$ to about $260 \mathrm{~K}$, respectively. ${ }^{24}$ Pressure is another external stimuli that can be used to tune the strength of the hydrogen bonds and tailor the properties of these compounds. For instance, it has been reported that the polar character of $\left[\mathrm{NH}_{4}\right]\left[\mathrm{Cd}(\mathrm{HCOO})_{3}\right]$ can be enhanced greatly by the application of pressure. ${ }^{25}$ The application of pressure may also lead to the discovery of new polymorphs that are not accessible under ambient conditions, as shown for a number of chiral and perovskite-like formates. $^{25-29}$

To date, only two dense perovskite-like MOFs containing imidazolium cations are known. The first, $(\mathrm{HIm})_{2}\left[\mathrm{KFe}(\mathrm{CN})_{6}\right]$, wherein $\mathrm{HIm}^{+}=$imidazolium cation, undergoes order-disorder phase transitions and exhibits striking dielectric anomalies and anisotropy below room temperature. ${ }^{30}$ The second one, [HIm]$\left[\mathrm{Mn}(\mathrm{HCOO})_{3}\right](\mathrm{ImMn})$, was discovered by Pato Dolán et al., who observed magnetic order below $9 \mathrm{~K}$ and interesting dielectric properties. $^{31}$ Based on the obtained results, it was suggested that this compound exhibits antiferroelectric order below $220 \mathrm{~K}$, i.e., ImMn shows the coexistence of magnetic and electric order in one phase. ${ }^{31}$ However, DSC did not reveal any anomaly indicative of a phase transition. ${ }^{31}$ Recently, a phase transition in ImMn was discovered at $438 \mathrm{~K}$ and it was suggested that the hightemperature phase has tetragonal symmetry. ${ }^{32}$

The abovementioned examples show that to better understand mechanism of the phase transition and structure-property relationship in metal formate frameworks, it is important to perform a temperature- and pressure-dependent study of these compounds using various experimental methods. Because in this family of formates, hydrogen bonds play significant role, it is especially important to employ experimental methods that are sensitive to amine-framework interactions, e.g., neutron diffraction, NMR, infrared (IR) and Raman spectroscopy. We have, therefore, decided to synthesise a previously unreported imidazolium magnesium formate (ImMg) and employ temperature-dependent IR and Raman spectroscopy, as well as pressure-dependent Raman spectroscopy of this compound as vibrational spectroscopy is a very sensitive probe for monitoring the changes in hydrogen bonds, dynamics of the molecular units and lattice distortion. ${ }^{33}$ For comparison, we also report temperature-dependent data for known ImMn that provides additional insight into the properties of this compound and mechanism of the phase transition.

\section{Experimental}

\section{Materials and instrumentation}

$\mathrm{MnCl}_{2}$ (99\%, Sigma-Aldrich), $\mathrm{MgCl}_{2} \cdot 6 \mathrm{H}_{2} \mathrm{O}$ (99-102\%, Sigma-Aldrich), methanol (99.8\%, Sigma-Aldrich), imidazole (99\%, Sigma-Aldrich) and formic acid (98\%, Fluka) were available commercially and used without further purification. The heat capacity was measured using Mettler Toledo DSC-1 calorimeter with a high resolution of $0.4 \mu \mathrm{W}$. Nitrogen was used as a purging gas. The weight of the ImMg (ImMn) sample was $32.1 \mathrm{mg}(21.4 \mathrm{mg})$. The heating and cooling rate was $5 \mathrm{~K} \mathrm{~min}^{-1}$. The excess heat capacity associated with the phase transition was evaluated by subtraction from the data the baseline representing the variation in the absence of the phase transitions. The dielectric measurements at ambient pressure were carried out using a Novocontrol Alpha impedance analyzer $(1 \mathrm{~Hz}-1 \mathrm{MHz})$. Because the obtained single crystals were not large enough to perform single-crystal dielectric measurements, pellets made of well-dried samples were measured instead. The pellets were placed between two flat copper electrodes of the capacitor with a gap of $0.4 \mathrm{~mm}$. The small signal of amplitude $1 \mathrm{~V}$ was applied across the sample. The temperature was controlled by the Novo-Control Quattro system, with a use of a nitrogen gas cryostat. The measurements were taken every $1 \mathrm{~K}$ over the temperature range from 390 to $475 \mathrm{~K}$. The temperature stability of the samples was better than $0.1 \mathrm{~K}$. The single-crystal X-ray diffraction experiment was carried out on an Xcalibur, Sapphire1 diffractometer operating in $\kappa$-geometry, equipped with a 2D CCD detector and a Mo $K \alpha$ radiation source $(\lambda=0.71073 \AA)$. For hightemperature X-ray diffraction, the sample was mounted in a quartz capillary. The high-temperature was provided with a hot-air flow (Kuma Diffraction). The X-ray powder diffraction data were collected using a X'Pert PRO X-ray diffraction system equipped with PIXcel ultra-fast line detector and Soller slits for $\mathrm{Cu} \mathrm{K}_{\alpha}$ radiation. The powders were closed in quartz capillaries (with $\phi=0.5 \mathrm{~mm}$ ) and measured in the transmission mode in the Anton Paar $1200 \mathrm{~N}$ High-Temperature Oven Chamber. The temperature-dependent Raman spectra were obtained using a Renishaw InVia Raman spectrometer equipped with confocal DM 2500 Leica optical microscope, a thermoelectrically cooled CCD as a detector, an argon laser operating at $488 \mathrm{~nm}$ and Linkam cryostat cell. The temperature-dependent IR spectra were obtained using a Biorad 575C FT-IR spectrometer and a home made furnace. The spectral resolution of the Raman and IR spectra was $2 \mathrm{~cm}^{-1}$. The highpressure Raman spectra were obtained in back-scattering geometry using a microscope attached to a triple-grating spectrometer Jobin Yvon T64000. The $514.5 \mathrm{~nm}$ line of an argon-ion laser was used as excitation and the spectral resolution was $2 \mathrm{~cm}^{-1}$. To reach high pressures, a diamond anvil cell $\mu$-scope DAC HT(S) from Almax easyLab with a diamond culet of $0.4 \mathrm{~mm}$ was used. The sample was loaded into a $100 \mu \mathrm{m}$ hole drilled in a stainless steel gasket with a thickness of $200 \mu \mathrm{m}$ using an electric discharge machine from Almax easyLab. Nujol served as the pressure transmitting media. Pressures were measured based on the shifts of the ruby R1 and R2 fluorescence lines.

\section{Synthesis of the samples}

To obtain single crystals of ImMg or ImMn, $16 \mathrm{~mL}$ of a methanol solution containing $5 \mathrm{mmol}$ of imidazole and $20 \mathrm{mmol}$ of formic acid was placed at the bottom of a glass tube $(9 \mathrm{~mm}$ inner diameter). To this solution, $16 \mathrm{~mL}$ methanol solution containing $2 \mathrm{mmol}$ of $\mathrm{MgCl}_{2} \cdot 6 \mathrm{H}_{2} \mathrm{O}$ or $\mathrm{MnCl}_{2}$ was added gently. The tube was 
sealed and kept undisturbed. Colorless (pale pink) transparent crystals of ImMg (ImMn) were harvested after 1 week.

\section{Crystallographic structure determination}

The data were collected in the $\omega$-scan mode with $\Delta \omega=1.0^{\circ}$ and processed with CrysAlis PRO, Agilent Technologies, Version 1.171.37.35h. The structure was solved by direct methods and refined with SHELXL2014/7. ${ }^{34}$ An empirical absorption correction using spherical harmonics was implemented. The hydrogen atoms were placed in the calculated positions and refined as riding atoms. Crystal data, data collection and the refinement results for ImMg are presented in Table S1 (ESI $\dagger$ ). The selected bonds and the hydrogen bond geometry are given in Tables S2 and S3 (ESI $\dagger$ ).

\section{Results and discussion}

\section{DSC}

The DSC measurements show that ImMg exhibits a phase transition at $451 \mathrm{~K}$ upon heating and $448 \mathrm{~K}$ upon cooling (Fig. S1, ESI $\dagger$ ). Fig. 1 shows that the heat capacity peak is asymmetric. Furthermore, the change in entropy at the phase transition is not sharp but $\Delta S$ decreases gradually with decreasing temperature and reaches almost zero at $410 \mathrm{~K}$ (Fig. 1). This type of behavior and small thermal hysteresis suggest that the phase transition is close to a second-order one.

The associated change in enthalpy $\Delta H$ and entropy $\Delta S$ is $\sim 322 \mathrm{~J} \mathrm{~mol}^{-1}$ and $\sim 0.73 \mathrm{~J} \mathrm{~mol}^{-1} \mathrm{~K}^{-1}$, respectively. Isostructural ImMn showed slightly smaller values (see Fig. S2, ESI $\dagger$ ). For an order-disorder transition, $\Delta S=R \ln (N)$, where $N$ is the number of sites for the disordered system. ${ }^{35}$ For $N=2, \Delta S$ should be $5.8 \mathrm{~J} \mathrm{~mol}^{-1} \mathrm{~K}^{-1}$. The experimental value of $\Delta S$ for ImMg is almost eight times smaller than that expected for the simplest orderdisorder transition with only two sites in the disordered phase. Similar behavior was reported for many related perovskite-like formats, such formamidinium manganese formate $^{19}$ and $\left[\left(\mathrm{CH}_{3}\right)_{2} \mathrm{NH}_{2}\right]\left[\mathrm{Na}_{0.5} \mathrm{Fe}_{0.5}(\mathrm{HCOO})_{3}\right],{ }^{36}$ which indicates that the phase transition is more complex than expected on the basis of a simple order-disorder model. In particular, the lower

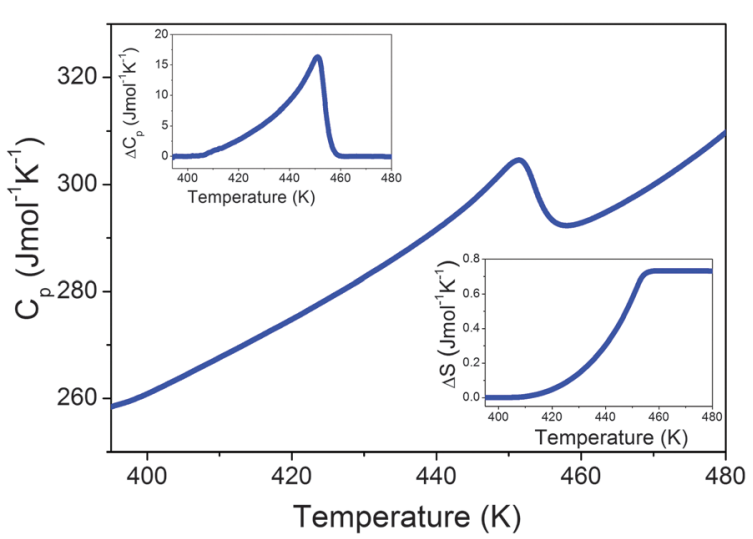

Fig. 1 Heat capacity of ImMg measured in a heating mode. The insets show the change in $C_{p}$ and $S$ related to the phase transition. than expected entropy change suggests that imidazolium cations are not fully ordered just below the phase transition, but still retain significant degrees of freedom well below $T_{\mathrm{c}}$.

\section{X-ray diffraction studies}

ImMg crystallizes in the monoclinic system in the $P 2_{1} / n$ space group with lattice parameters $a=12.1246(4) \AA, b=12.2087(5) \AA$, $c=12.4991(4) \AA, \beta=91.39(1)^{\circ}$ at $296 \mathrm{~K}$ (Table S1, ESI $\dagger$ ). The space group is different from that reported by Wang et al. ${ }^{32}$ $\left(P 2_{1} / c\right)$, but it is in agreement with the crystal structure of ImMn reported by Pato Dolán et al. ${ }^{31}$ Therefore, ImMg is isostructural with ImMn; however, its lattice parameters are slightly smaller compared to ImMn $(a=12.3034(4) \AA, \quad b=12.4408(4) \AA$, $c=12.8156(4) \AA, \beta=91.190(2)^{\circ}$ at $\left.295 \mathrm{~K}^{31}\right)$. The $\mathrm{Mg}(\mathrm{HCOO})_{3}{ }^{-}$ ions form a perovskite-like architecture, which accommodates $\mathrm{HIm}^{+}$templates. There are two independent imidazolium cations in the asymmetric unit. Both interact with the $\mathrm{Mg}$-formate framework through the $\mathrm{N}-\mathrm{H} \cdots \mathrm{O}$ and $\mathrm{C}-\mathrm{H} \cdots \mathrm{O}$ hydrogen bonds of a medium strength. The donor to acceptor distances in the $\mathrm{N}-\mathrm{H} \cdots \mathrm{O}$ bonds range from $2.751(3)$ to $2.873(3) \AA$; the $\mathrm{C}-\mathrm{H} \cdots \mathrm{O}$ interactions are weaker, with donor to acceptor distances of 3.111(4) and 3.176(4) A (Table S3, ESI $\dagger$ ). Similar N . . O distances, ranging from $2.779(2)$ to $2.846(2) \AA$, were reported for ImMn. ${ }^{31}$ The imidazolium cations are prone to temperature-induced librations and rotations. ${ }^{37,38}$ Herein, the hydrogen bonds are strong enough to preserve the rotations. Fig. S3 (ESI $\dagger$ ) shows both imidazolium cations in the ellipsoid representation and their placement in the crystal cavities. Large displacement parameters at room temperature indicate the presence of a substantial motion, wherein the most expected are the in-plane librations. In both ImMg and ImMn, the basal planes of the $\mathrm{HIm}^{+}$are situated nearly parallel to the (111) and $(-111)$ planes of the perovskite-like cell. The symmetry center results in the antiparallel arrangement of the dipole moments associated with each imidazolium cation. In addition, two inequivalent $\mathrm{A}$ and $\mathrm{B}$ cations form two substructures spontaneously polarized in the antiparallel directions. Pato Dolan et al. postulated the antiferroelectric character of ImMn based on the arrangement of the imidazolium dipole moments. ${ }^{31}$ This study provides additional evidence supporting the antiferroelectric character of the low-temperature phase in both crystals.

Fig. 2 illustrates the distribution of the $\mathrm{HIm}^{+}$dipole moments in the ImMg at room temperature. In this compound, the phase transition at $T_{\mathrm{c}}=451 \mathrm{~K}$ is associated with a reduction of the unit cell size. Fig. S4 (ESI $\dagger$ ) presents the reciprocal lattice of ImMg at room temperature and at $460 \mathrm{~K}$. At $460 \mathrm{~K}$, the $h k l$ layers with $l=2 n+1$ vanish, indicating a halving of the $c$ lattice parameter. The monoclinic symmetry is preserved and the new metrics are: $a=12.261(7) \AA, b=12.290(4) \AA, c=6.280(4) \AA, \beta=$ $90.62(5)^{\circ}$. The reduction of the unit cell size destroys the antiferroelectric order of the dipoles in both sublattices. Herein, we have to add that the complete analysis of the crystal structure of ImMg at $460 \mathrm{~K}$ was not possible due to the rapid decomposition of the single-crystals, even though they were protected in the capillary. Nevertheless, the data collected were sufficient to define without any doubts the metrics of the new phase. This was additionally confirmed by high-temperature 


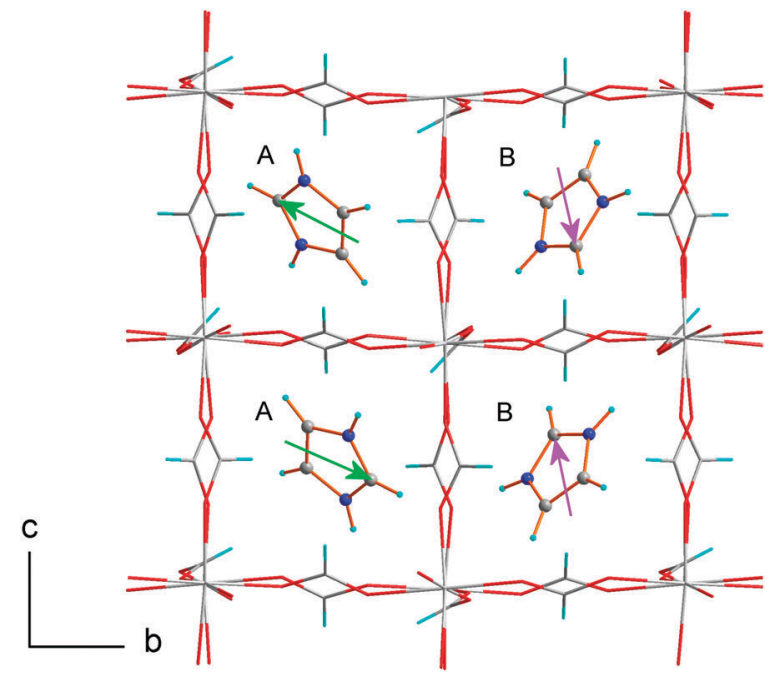

Fig. $21 / 2 a, b$, c unit cell of ImMg at room temperature. Along the $c$ axis, there is an antiparallel setting of the $\mathrm{HIm}^{+}$dipole moments. The $\mathrm{A}$ and $\mathrm{B}$ mark two inequivalent substructures.

powder X-ray diffraction. Fig. S6-S8 (ESI $\dagger$ ) present diffractograms of ImMg and ImMn measured at high temperatures. La Bail fits in both phases of both crystals were carried out using the monoclinic unit cells. The most evident difference between the high- and low-temperature patterns detected for ImMg and ImMn is the vanishing of the $(-1-11),(-111),(1-11)$ and (111) diffraction peaks after the phase transition, signifying the reduction of the unit cell size.

These findings stay opposite to the recently published data for ImMn. ${ }^{32}$ Wang et al. postulated that at $T_{\mathrm{c}}=438 \mathrm{~K}$ symmetry increases from monoclinic to tetragonal. However, their tetragonal cell choice skips a number of measured diffraction peaks and the lattice parameters, especially the angles, depart significantly from the tetragonal ones, observe Fig. S5 (ESI $\dagger$ ). Furthermore, the calculated powder diagram based on the tetragonal structure differs substantially from the measured data presented both in this study and by Wang et al. ${ }^{32}$ Finally, the experimental powder diffractograms presented by Wang et al. for the hightemperature phase of ImMn at 443, 453 and $463 \mathrm{~K}$ show the presence of strong peaks characteristic of the decomposed sample (see Wang et al. $^{32}$ and our diffractogram of ImMn measured at $503 \mathrm{~K}$, Fig. S7, ESI $\dagger$ ).

\section{Dielectric measurements}

Fig. 3 shows that the temperature dependence of the dielectric permittivity for ImMg and ImMn are similar, i.e., $\varepsilon^{\prime}$ decreases with increasing frequency at all temperatures and this type of behavior is a characteristic feature of polar dielectric materials. The rapid increase in the imaginary part of the dielectric permittivity with temperature clearly confirms that temperature starts to activate conductivity in the investigated samples. The plots of $\varepsilon^{\prime} v s$. temperature indicate the broad dielectric anomalies in the vicinity of the phase transition temperatures. The strong frequency dispersion of these anomalies implies a relaxor nature of electric ordering in the investigated samples. An observation of
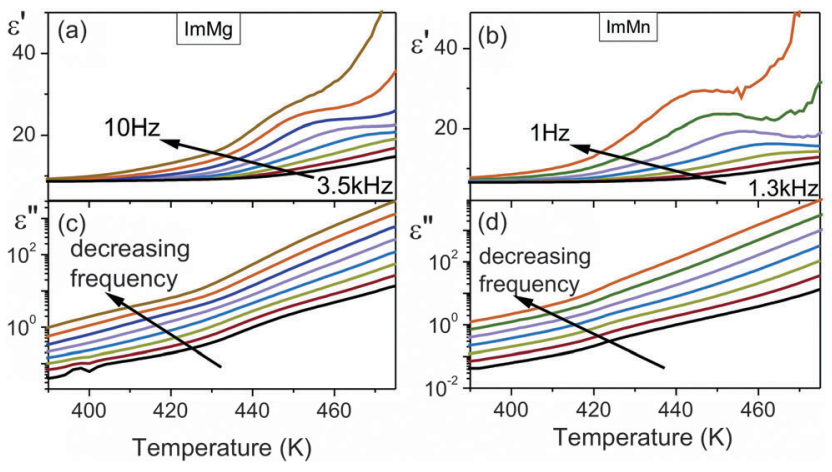

Fig. 3 Temperature dependence of the real $\varepsilon^{\prime}$ part of the dielectric permittivity for (a) ImMg and (b) ImMnMn. Temperature dependence of the imaginary $\varepsilon^{\prime \prime}$ part for $I \mathrm{mMg}$ and $I \mathrm{mMn}$ are shown in (c) and (d), respectively.

a similar broad peak in $\varepsilon^{\prime}$ was recently reported for ImMn by Wang et al. , but they did not observe a conductivity process. ${ }^{32}$ It is worth noting, however, that conductivity above room temperature is often observed for compounds containing the imidazole group. ${ }^{39}$

Based on the values of the activation energy and the relaxation times, it is possible to find a reason of the observed processes. To distinguish the local dielectric relaxation from long-range electrical conductivity in the investigated samples, a frequency domain was used. The data obtained from impedance spectroscopy technique can be analyzed in terms of four equivalent formalisms: electric modulus $M^{*}$, impedance $Z^{*}$, admittance $Y^{*}$, and permittivity $\varepsilon^{*}$. The observed conductivity process can be well observed when the data are presented in the electric modulus representation. ${ }^{40}$ The advantage of this approach over the classical impedance Bode plots formalism is that $M^{*}$ diminishes the electrode capacitance effects and simply allows for the conductivity process analysis. ${ }^{41}$ The modulus spectra $M^{\prime}$ and $M^{\prime \prime}$ for ImMg and ImMn are depicted in Fig. 4. The imaginary part of the electric modulus exhibits a characteristic peak, which corresponds to the so-called conductivity relaxation that moves towards higher frequencies during heating. The peaks are broader and more asymmetric rather than symmetric, as predicted by an ideal Debye behavior.

The relaxation times, $\tau\left(=1 / \omega_{\max }\right)$, obtained from the maximum of $M^{\prime \prime}$ at various temperatures, are presented in Fig. 5. The relaxation times can be characterized by approximately two linear
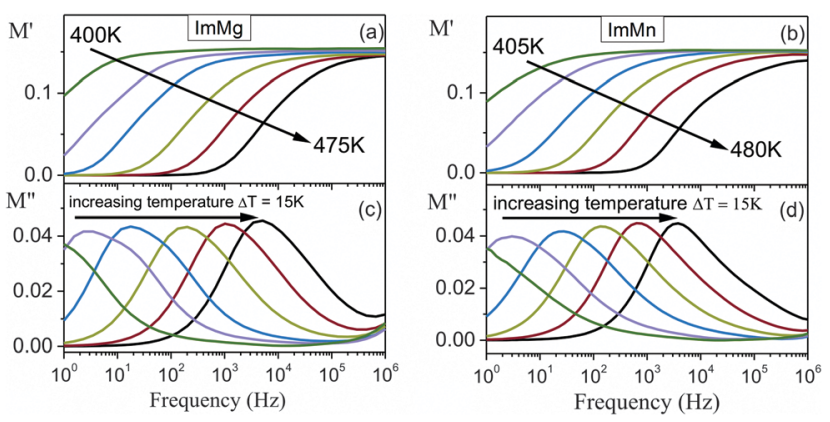

Fig. 4 Frequency dependence of the real $M^{\prime}$ and imaginary $M^{\prime \prime}$ parts of the electric modulus for $\operatorname{ImMg}$ ( $a$ and c) and ImMn (b and d). 

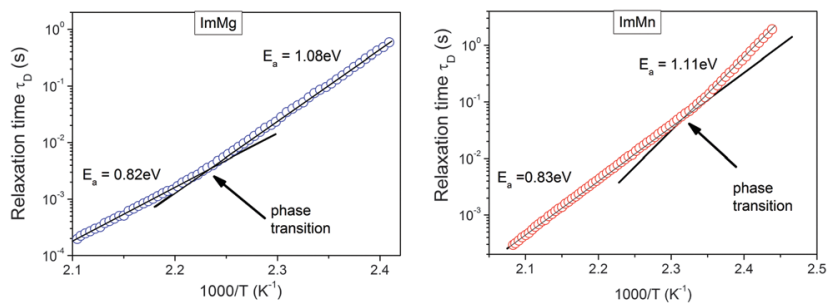

Fig. 5 Arrhenius plot of the relaxation times for observed conductivity relaxation processes observed in the modulus spectra. The arrows indicate the phase transition temperatures.

functions, indicating two different thermally activated processes, which dominate the conductivity below, and above the phase transition. Thus, the Arrhenius law can be used to describe these data. The activation energies obtained are characteristic of the imidazole-based compounds, wherein the observed long-range conduction is explained by the ion jumping through defect sites. $^{42}$

\section{Temperature-dependent Raman and IR studies}

The temperature-dependent Raman and IR spectra of ImMg are presented in Fig. 6 and Fig. S9, S10 (ESI $\dagger$ ). Table S4 (ESI $\dagger$ ) lists the wavenumber of the observed modes together with the proposed assignment based on the vibrational data reported for the related formates, as well as imidazolium cation, 4-methylimidazolium cation and imidazolium salts. ${ }^{9,13,19,43-46}$

Raman and IR spectra of ImMg show a few characteristic changes upon heating. First, the intensity of many bands and shoulders decrease continuously upon heating and disappear at higher temperatures. This behavior is observed for the Raman bands at 3126, 2877, 1440, 1376 and $1382 \mathrm{~cm}^{-1}$, as well as IR bands at 1459, 1115, 911 and $861 \mathrm{~cm}^{-1}$ (see Fig. 6 and Fig. S9, S10, ESI $\dagger$ ). Furthermore, the 1114 and $1093 \mathrm{~cm}^{-1}$ Raman bands coalescence into one broad band at $1098 \mathrm{~cm}^{-1}$ (see Fig. 6 and Fig. S11b, ESI $\dagger$ ). It is worth noting that all abovementioned IR bands and most of the Raman bands correspond to vibrations of

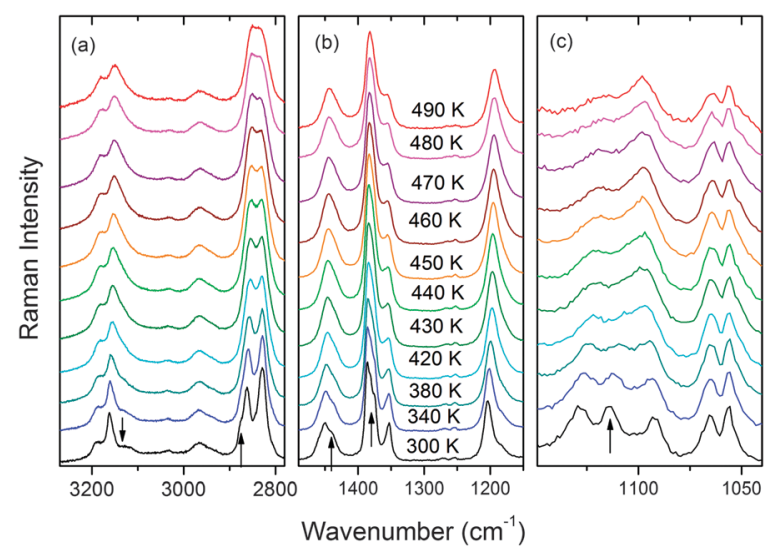

Fig. 6 Raman spectra of ImMg corresponding to the spectral ranges of $2780-3270,1150-1490$ and $1040-1150 \mathrm{~cm}^{-1}$ at different temperatures in a heating run. The arrows indicate the bands that disappear as a result of the phase transition. the imidazolium cation. The decrease in the number of these bands above $T_{\mathrm{c}}$ indicates that the number of crystallographically distinct $\mathrm{HIm}^{+}$cations changes from two in the low-temperature phase to only one in the high-temperature phase. In contrast to the bands of $\mathrm{HIm}^{+}$cations, Raman and IR bands, corresponding to $\mathrm{HCOO}^{-}$ions, show very weak changes. The vibrational data are, therefore, consistent with a halving of the unit cell size and a preservation of the monoclinic symmetry, indicated by the X-ray diffraction data.

Raman and IR spectra also show that some bands exhibit pronounced broadening upon heating (Fig. 6 and Fig. S11c, ESI $\dagger$ ). This behavior is especially pronounced for the IR (Raman) bands observed in the regions of 820-1150, 1440-1460 and 3110$3200 \mathrm{~cm}^{-1}$ (1090-1130 and 3110-3200 $\mathrm{cm}^{-1}$ ), which correspond to vibrations of the imidazolium cation, and lattice modes observed below $250 \mathrm{~cm}^{-1}$ (Fig. 6 and Fig. S9, S10, ESI $\dagger$ ). This behavior proves that an increase in temperature leads to strong activation of the molecular motions of the whole imidazolium cation and the phase transition is associated with the disordering of $\mathrm{HIm}^{+}$cations.

Raman data for ImMn (Fig. S12 and S13, ESI $\dagger$ ) show that the observed changes upon heating are very similar to those observed for ImMg. The IR spectra of ImMn reported by Wang et $a .^{33}$ also show similar changes upon heating as the ImMg spectra. This behavior points to the same phase transition mechanism for both compounds.

\section{Pressure-dependent Raman scattering studies}

When the pressure increases, the spectra remain similar up to 2.3 GPa (Fig. 7). At 3.7 GPa, the shoulders become evident at 239 and $1237 \mathrm{~cm}^{-1}$. Furthermore, the weak band at $1121 \mathrm{~cm}^{-1}$ disappears, the 632 and $640 \mathrm{~cm}^{-1}$ exhibit a clear upshift by about $5 \mathrm{~cm}^{-1}$, the $139 \mathrm{~cm}^{-1}$ band shifts to lower wavenumber and the $174 \mathrm{~cm}^{-1}$ band (at $2.3 \mathrm{GPa}$ ) becomes broad and asymmetric at 3.7 and 5.1 GPa. Many modes also exhibit significant changes in the slope of the wavenumber $v s$. pressure (Fig. S14 and Table S5, ESI $\dagger$ ). These changes suggest that ImMg exhibits some subtle

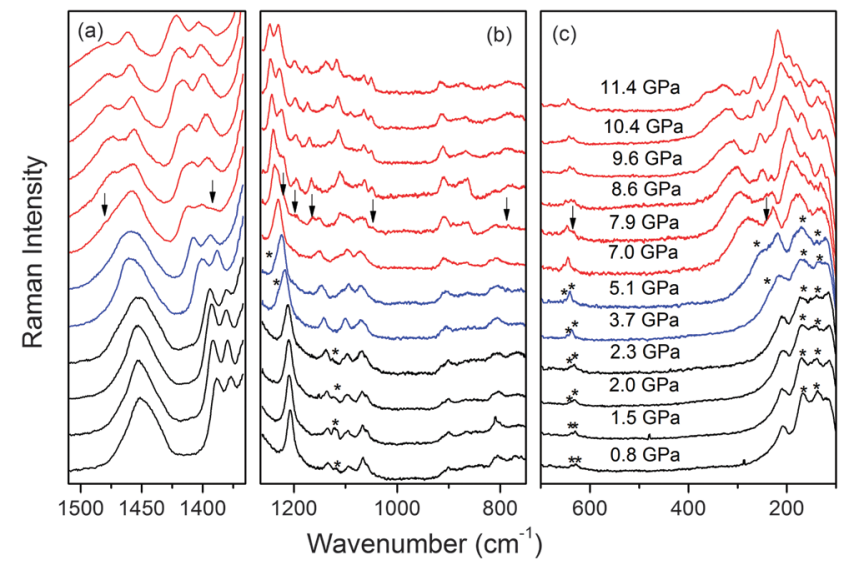

Fig. 7 Pressure-dependent Raman spectra of ImMg obtained during the compression experiment. The asterisks denote the bands that appear, disappear and shift due to the phase transition between 2.3 and $3.7 \mathrm{GPa}$ (see discussion). The arrows indicate bands that appear at 7.0 and $7.9 \mathrm{GPa}$. 
structural change between 2.3 and 3.7 GPa. Because the discussed bands correspond to vibrations of the imidazolium cation and the lattice modes, this transition is most likely associated with a rearrangement of the imidazolium cations without any significant distortion of these cations and the magnesium formate framework.

When the pressure increases to $7 \mathrm{GPa}$, new shoulders and weak bands appear at 242, 1389 and $1482 \mathrm{~cm}^{-1}$ (see Fig. 7). Their intensity strongly increases at $7.9 \mathrm{GPa}$ and a number of other bands appear at 631, 786, 1048, 1164, 1195 and $1220 \mathrm{~cm}^{-1}$ (Fig. 7 and Fig. S14, ESI $\dagger$ ). Many narrow bands appear also below $200 \mathrm{~cm}^{-1}$. The intensity of the new bands further increases upon compression to 8.6 GPa. The appearance of Raman bands is associated with the disappearance or strong decrease in intensity of the $218,1094,1147,1241,1408 \mathrm{~cm}^{-1}$ bands (values at $5.1 \mathrm{GPa}$, Fig. 7 and Fig. S14, ESI $\dagger$ ). These changes indicate that ImMg starts to transform to a new high-pressure phase when pressure is close to $7.0 \mathrm{GPa}$ and when the pressure reaches $8.6 \mathrm{GPa}$, this transformation is complete.

The observed changes in the Raman spectra allow us to obtain a few conclusions on structural changes occurring upon compression. First, the pressure coefficients $\alpha$ of the ambient pressure and intermediate phases are 2.13-4.57 and 0.36$0.86 \mathrm{~cm}^{-1} \mathrm{GPa}^{-1}$ for the bands near $1380-1420$ and $800 \mathrm{~cm}^{-1}$, related to the $\nu_{5}$ and $\nu_{3}$ modes of $\mathrm{HCOO}^{-}$, respectively (Table S5, ESI $\dagger)$. Because the $\nu_{5}\left(\nu_{3}\right)$ modes correspond to $\mathrm{C}-\mathrm{H}(\mathrm{O}-\mathrm{C}-\mathrm{O})$ vibrations, ${ }^{13}$ their strong (weak) pressure dependence indicates that the $\mathrm{C}-\mathrm{H}(\mathrm{C}-\mathrm{O})$ bond lengths significantly (weakly) decrease upon compression up to $7 \mathrm{GPa}$. The $\mathrm{C}-\mathrm{O}$ bonds are, however, strongly affected by the phase transition near $7 \mathrm{GPa}$, as evidenced by strong pressure dependence and splitting of the $\nu_{3}$ mode above $7 \mathrm{GPa}$. Second, doubling of the bands related to the $\nu_{5}$ and $\nu_{3}$ modes of $\mathrm{HCOO}^{-}$points to a doubling of the crystallographically unique formate ions. Therefore, the phase transition is associated with significant distortion of the metal formate framework. Third, the phase transition near $7 \mathrm{GPa}$ leads to a strong increase in the number of bands related to the imidazolium cations (see region $1050-1250 \mathrm{~cm}^{-1}$ in Fig. 7). This behavior indicates a lowering of the crystal symmetry and an increase in the number of crystallographically unique imidazolium cations. Previous studies of related azetidinium zinc formate (AzZn) showed that the conformation of the azetidinium cation is strongly affected by the phase transition near about $2.4 \mathrm{GPa}^{28}$ Similarly, the structure of the protonated amine was strongly affected in dimethylammonium manganese formate (DMMg) upon the transformation near $5.6 \mathrm{GPa} .{ }^{29} \mathrm{ImMg}$ shows this effect at higher pressures, i.e. close to $7 \mathrm{GPa}$, which suggests that its metal formate framework is stiffer than the frameworks of the mentioned above compounds. Fourth, imidazolium bands in the range of $1050-1250 \mathrm{~cm}^{-1}$ exhibit significant narrowing above $7 \mathrm{GPa}$. Furthermore, the Raman spectra do not show a decrease in intensity even at $11.4 \mathrm{GPa}$. This indicates that the imidazolium cations are well ordered in the high-pressure phase. Very different behavior was reported for $\left[\mathrm{ND}_{4}\right]\left[\mathrm{Zn}(\mathrm{DCOO})_{3}\right]$, AzZn and $\mathrm{DMMg}$, for which strong broadening and a decrease in intensity of the Raman bands, which is indicative of partial amorphization, was observed close to $2.8,7.4$ and $6.8 \mathrm{GPa}$, respectively. ${ }^{26,28,29}$

The Raman spectra of ImMg during the decompression are presented in Fig. S14 (ESI $\dagger$ ). The observed changes in the Raman spectra are similar to those observed upon compression, indicating reversibility of the phase transitions.

\section{Conclusions}

We report the synthesis and characterization of ImMg perovskite-type formate. This compound crystallizes in the monoclinic structure with an antiparallel arrangement of the dipole moments associated with two inequivalent imidazolium cations. DSC and detailed temperature-dependent X-ray diffraction, dielectric, Raman and IR data show that this compound undergoes a structural phase transition at $451 \mathrm{~K}$, the highest temperature found for any metal formate framework. Although the crystal structure of the high-temperature phase could not be solved due to the poor chemical stability of this compound above $450 \mathrm{~K}, \mathrm{X}$-ray diffraction provides evidence that the monoclinic symmetry is preserved above $T_{\mathrm{c}}$, but the $c$ parameter is halved and the antiferroelectric order of the dipoles is destroyed. The Raman and IR data are consistent with the weak modification of the framework and halving of the unit-cell volume of the hightemperature phase. They also provide evidence that an increase in temperature leads to the strong activation of molecular motion of the imidazolium cation, i.e., order-disorder processes may contribute significantly to the phase transition mechanism. The dielectric data show that ImMg exhibits relaxor type of behavior and significant ionic conductivity. It is worth adding that our data also indicate the same phase transition mechanism for the ImMn compound. However, the X-ray diffraction and vibrational data are not consistent with those reported by Wang et al., ${ }^{32}$ i.e., an increase in symmetry to tetragonal.

ImMg also exhibits very interesting behavior under hydrostatic compression. First, its Raman spectra remain very intense and the Raman bands are narrow up to the highest pressure reached in these experiments, i.e., up to $11.4 \mathrm{GPa}$. This behavior indicates that the magnesium formate framework does not collapse and the long-range order is well preserved, even at very high pressures. This behavior is quite different from that reported previously for metal formates templated by ammonium, azetidinium and dimethylammonium cations, which showed signs of significant disorder or even partial amorphization well below $10 \mathrm{GPa}^{26,28,29}$ Despite the strong stability of the ImMg framework, this compound undergoes two pressureinduced phase transitions near about 3 and $7 \mathrm{GPa}$. The first transition leads to subtle changes in the crystal structure; however, the second one leads to strong distortion of the magnesium formate framework.

\section{Acknowledgements}

The authors acknowledge the Brazilian National Research Council (CNPq) for a fellowship and the grant 401849/2013-9. 


\section{References}

1 R. J. Kuppler, D. J. Timmons, Q.-R. Fang, J.-R. Li, T. A. Makal, M. D. Young, D. Yuan, D. Zhao, W. Zhuang and H.-C. Zhou, Coord. Chem. Rev., 2009, 253, 3042-3066.

2 R. Shang, S. Chen, Z. M. Wang and S. Gao, in Metal-Organic Framework Materials, ed. R. L. MacGillivray and C. M. Lukehart, John Wiley \& Sons Ltd, 2014, pp. 221-238.

3 M. Guo, H.-L. Cai and R.-G. Xiong, Inorg. Chem. Commun., 2010, 13, 1590-1598.

4 Z.-Y. Du, T.-T. Xu, B. Huang, Y.-J. Su, W. Xue, C.-T. He, W.-X. Zhang and X.-M. Chen, Angew. Chem., Int. Ed., 2015, 54, 914-918.

5 (a) W. Zhang, H.-Y. Ye, R. Graf, H. W. Spiess, Y.-F. Yao, R.-Q. Zhu and R.-G. Xiong, J. Am. Chem. Soc., 2013, 135, 5230-5233; (b) W.-J. Xu, S.-L. Chen, Z.-T. Hu, R.-B. Lin, Y.-J. Su, W.-X. Zhang and X.-M. Chen, Dalton Trans., 2015, 45, 4224-4229.

6 J. M. Bermúdez-García, M. Sánchez-Andújar, S. Yáñez-Vilar, S. Castro-Garcia, R. Artiaga, J. López-Beceiro, L. Botana, A. Alegría and M. A. Señaris-Rodriguez, Inorg. Chem., 2015, 54, 11680-11687.

7 (a) P. Jain, V. Ramachandran, R. J. Clark, H. D. Zhou, B. H. Toby, N. S. Dalal, H. W. Kroto and A. K. J. Cheetham, J. Am. Chem. Soc., 2009, 131, 13625-13627; (b) M. SánchezAndújar, S. Presedo, S. Yáñez-Vilar, S. Castro-Garcia, J. Shamir and M. A. Señaris-Rodriguez, Inorg. Chem., 2010, 49, 1510-1516; (c) W. Wang, L.-Q. Yan, J.-Z. Cong, Y.-L. Zhao, F. Wang, S.-P. Shen, T. Zhou, D. Zhang, S.-G. Wang, X.-F. Han and Y. Sun, Sci. Rep., 2013, 3, 2024.

8 (a) Y. Tian, J. Cong, S. Shen, Y. Chai, L. Yan, S. Wang and Y. Sun, Phys. Status Solidi RRL, 2014, 8, 91-94; (b) Y. Tian, A. Stroppa, Y. Chai, L. Yan, S. Wang, P. Barone, S. Picozzi and Y. Sun, Sci. Rep., 2014, 4, 6062.

9 (a) M. Mączka, A. Gạgor, B. Macalik, A. Pikul, M. Ptak and J. Hanuza, Inorg. Chem., 2014, 53, 457-467; (b) M. Maczka, M. Ptak and L. Macalik, Vib. Spectrosc., 2014, 71, 98-104.

10 M. Šimenas, A. Ciupa, M. Mạczka, A. Pöppl and J. Banys, J. Phys. Chem. C, 2015, 119, 24522-24528.

11 (a) M. Kosa and D. T. Major, CrystEngComm, 2015, 17, 295-298; (b) G. P. Nagabhushana, R. Shivaramaiah and A. Navrotsky, J. Am. Chem. Soc., 2015, 137, 10351-10356.

12 (a) G. C. Xu, W. Zhang, X. M. Ma, Y. H. Hen, L. Zhang, H. L. Cai, Z. M. Wang, R. G. Xiong and S. J. Gao, J. Am. Chem. Soc., 2011, 133, 14948-14951; (b) J. M. M. Lawler, P. Manuel, A. L. Thompson and P. J. Saines, Dalton Trans., 2015, 44, 11613-11620.

13 (a) M. Mączka, A. Pietraszko, B. Macalik and K. Hermanowicz, Inorg. Chem., 2014, 53, 787-794; (b) M. Maczka, K. SzymborskaMałek, A. Ciupa and J. Hanuza, Vib. Spectrosc., 2015, 77, 17-24.

14 (a) J. Xu, B. E. G. Lucier, R. Sinelnikov, V. V. Terskikh, V. N. Staroverov and Y. Huang, Chem. - Eur. J., 2015, 21, 14348-14361; (b) M. Macczka, M. Ptak and S. Kojima, Appl. Phys. Lett., 2014, 104, 222903.

15 S. Chen, R. Shang, K.-L. Hu, Z.-M. Wang and S. Gao, Inorg. Chem. Front., 2014, 1, 83-98.
16 S. Chen, R. Shang, B.-W. Wang, Z.-M. Wang and S. Gao, Angew. Chem., Int. Ed., 2015, 54, 11093-11096.

17 (a) G. Kieslich, S. Kumagai, K. T. Butler, T. Okamura, C. H. Hendon, S. Sun, M. Yamashita, A. Walsh and A. C. Cheetham, Chem. Commun., 2015, 51, 15538-15541; (b) G. Kieslich, A. C. Forse, S. Sun, K. T. Butler, S. Kumagai, Y. Wu, M. R. Warren, A. Walsh, C. P. Grey and A. C. Cheetham, Chem. Mater., 2016, 28, 312-317.

18 (a) A. Stroppa, P. Barone, P. Jain, J. M. Perez-Mato and S. Picozzi, Adv. Mater., 2013, 25, 2284-2290; (b) D. Di Sante, A. Stroppa, P. Jain and S. Picozzi, J. Am. Chem. Soc., 2013, 135, 18126-18130.

19 M. Mączka, A. Ciupa, A. Gągor, A. Sieradzki, A. Pikul, B. Macalik and M. Drozd, Inorg. Chem., 2014, 53, 5260-5268.

20 (a) L. Cañadillas-Delgado, O. Fabelo, J. A. RodríguezVelamazán, M.-H. Lemėe-Cailleau, S. A. Mason, E. Pardo, F. Lloret, J.-P. Zhao, X.-H. Bu, V. Simonet, C. V. Colin and J. Rodríguez-Carvajal, J. Am. Chem. Soc., 2012, 134, 19772-19781; (b) A. Ciupa, M. Maczzka, A. Gągor, A. Sieradzki, J. Trzmiel, A. Pikul and M. Ptak, Dalton Trans., 2015, 44, 8846-8854.

21 M. Mączka, A. Sieradzki, B. Bondzior, P. Dereń, J. Hanuza and K. Hermanowicz, J. Mater. Chem. C, 2015, 3, 9337-9345.

22 W. Li, Z. Zhang, E. G. Bithell, A. S. Batsanov, P. T. Barton, P. J. Saines, P. Jain, C. J. Howard, M. A. Carpenter and A. K. Cheetham, Acta Mater., 2013, 61, 4928-4938.

23 W. Li, M. R. Probert, M. Kosa, T. D. Bennett, A. Thirumurugan, R. P. Burwood, M. Parinello, J. A. K. Howard and A. K. Cheetham, J. Am. Chem. Soc., 2012, 134, 11940-11943.

24 B. Pato Dolán, M. Sánchez-Andújar, L. C. Gómez-Aguirre, S. Yáñez-Vilar, J. López-Beceiro, C. Garcia-Fernández, A. A. Haghighirad, F. Ritter, S. Castro-Garcia and M. A. Señaris-Rodriguez, Phys. Chem. Chem. Phys., 2012, 14, 8498-8501.

25 L. C. Gómez-Aguirre, B. Pato Dolán, A. Stroppa, S. YáñezVilar, L. Bayarjargal, B. Winkler, S. Castro-Garcia, J. Mira, M. Sánchez-Andújar and M. A. Señaris-Rodriguez, Inorg. Chem., 2015, 54, 2109-2116.

26 M. Mączka, P. Kadłubański, P. T. C. Freire, B. Macalik, W. Paraguassu, K. Hermanowicz and J. Hanuza, Inorg. Chem., 2014, 53, 9615-9624.

27 W. Li, A. Thirumurugan, P. T. Barton, Z. Lin, S. Henke, H. H. M. Yeung, M. T. Wharmby, E. G. Bithell, J. A. K. Howard and A. K. Cheetham, J. Am. Chem. Soc., 2014, 136, 7801-7804.

28 M. Mączka, T. Almeida da Silva, W. Paraguassu, M. Ptak and K. Hermanowicz, Inorg. Chem., 2014, 53, 12650-12657.

29 M. Maczka, T. Almeida da Silva, W. Paraguassu and K. Pereira da Silva, Spectrochim. Acta, Part A, 2016, 156, 112-117.

30 W. Zhang, Y. Cai, R.-G. Xiong, H. Yoshikawa and K. Awaga, Angew. Chem., Int. Ed., 2010, 49, 6608-6610.

31 B. Pato Dolán, L. C. Gómez-Aguirre, J. M. Bermúdez-García, M. Sánchez-Andújar, A. Fondado, J. Mira, S. Castro-Garcia and M. A. Señaris-Rodriguez, RSC Adv., 2013, 3, 22404-22411.

32 F.-F. Wang, C. Chen, Y. Zhang, H.-Y. Ye, Q. Ye and D.-W. Fu, J. Mater. Chem. C, 2015, 3, 6350-6358. 
33 (a) C. Carabatos-Nedelec and P. Becker, J. Raman Spectrosc., 1997, 28, 663-671; (b) M. Maczka, A. G. Souza Filho, W. Paraguassu, P. T. C. Freire, J. Mendes Filho and J. Hanuza, Prog. Mater. Sci., 2012, 57, 1335-1381.

34 G. M. Sheldrick, Acta Crystallogr., Sect. A: Found. Crystallogr., 2008, 64, 112-122.

35 (a) M. E. Lines and A. M. Glass, Principles and Applications of Ferroelectrics and Related Materials, Oxford University Press, 2001; (b) K. Moriya, T. Matsuo, H. Suga and S. Seki, Bull. Chem. Soc. Jpn., 1979, 52, 3152-3162.

36 M. Maczzka, A. Pietraszko, L. Macalik, A. Sieradzki, J. Trzmiel and A. Pikul, Dalton Trans., 2014, 43, 17075-17084.

37 A. Gagor, A. Piecha, R. Jakubas and A. Miniewicz, Chem. Phys. Lett., 2011, 503, 134-138.

38 M. Wẹcławik, A. Gạgor, A. Piecha, R. Jakubas and W. Medycki, CrystEngComm, 2013, 15, 5633-5640.
39 S. Liu, Z. Yue and Y. Liu, Dalton Trans., 2015, 44, 12976-12980.

40 A. Molak, M. Paluch, S. Pawlus, J. Klimontko, Z. Ujma and I. Gruszka, J. Phys. D: Appl. Phys., 2005, 38, 1450-1460.

41 J. C. Dyre, J. Appl. Phys., 1988, 64, 2456-2468.

42 V. Tricoli, G. Orsini and M. Anselmi, Phys. Chem. Chem. Phys., 2012, 14, 10979-10986.

43 K. Hasegawa, T. Ono and T. Noguchi, J. Phys. Chem. B, 2000, 104, 4253-4265.

44 A. A. Adesokan, G. M. Chaban, O. Dopfer and R. B. Gerber, J. Phys. Chem. A, 2007, 111, 7374-7381.

45 A. Piecha, R. Jakubas, G. Bator and J. Baran, Vib. Spectrosc., 2009, 51, 226-237.

46 K. Hołderna-Natkaniec, R. Jakubas and I. Natkaniec, Internal Dynamics of the Ferroelectric $\left(\mathrm{C}_{3} \mathrm{~N}_{2} \mathrm{H}_{5}\right)_{5} \mathrm{Bi}_{2} \mathrm{Cl}_{11}$ Studied by ${ }^{1} \mathrm{H}$ NMR and IINS Methods, in Ferroelectrics - Characterization and Modeling, ed. M. Lallart, Intech, 2011, pp. 41-60. 